\title{
Katastrophenereignisse an Hängen
}

\section{Der Hang}

Die konstituierenden Bestandteile einer Berglandschaft sind Hänge: Ein Tal ist durch Hänge begrenzt. Das Vorhandense in von Hängen definiert recht eigentlich die "Bergwelt"; - in einer Ebene gibt es keine Hänge.

Der alpine Lebensraum ist eine Berglandschaft. Alle mechanischen Ereignisse an den Hängen beeinflussen wesentlich die Nutzbarkeit des Raumes. Eine Kenntnis und ein Verständnis dieser Ereignisse, - besonders inwieweit sie durch menschliche Eingriffe beeinflusst werden (oder werden können), sind daher für den Umweltschutz von grundlegender Bedeutung.

\section{Stabilität der Hänge}

Durch ihre Neigung sind "Hänge" im Prinzip gravi tationell unstabile Gebilde. Sie bleiben nur dank der Materialfestigkeit über längere oder kürzere Zeitspannen bestehen. Aber auch während ihrer scheinbaren Stabilitätsperiode sind Hänge gewöhnlich langsamen Bewegungen unterworfen. Man nennt solche Bewegungen "Hangkriechen" oder "Talzuschub".

Wenn sich die Steilheit eines Hanges durch langsame Bewegungen, sei es durch Erosion durch einen Bach am Fuss, sei es durch tektonische Einflüsse vergrössert, kann es zu einem plötzlichen Ereignis kommen. Es mögen einzelne Felsbrocken herunterfallen (vor allem, wenn der "Hang" eine steile Wand ist), es können Teile des Hanges in sich zusammenbrechen und einen Schuttstrom auslösen; oder es kann gar der Hang in sich zusammenbrechen und zu einem Bergsturz Anlass geben. Schliesslich können äussere Einflüsse am Hang mechanische Ereignisse auslösen: Es können sich Schnee- und Eislawinen, sowie durch starke Regengüsse bedingte Vermurungen ergeben.

\section{Langsame Bewegungen}

Die langsamen Bewegungen an Hängen äussern sich in Hangkriechen, Rasengleiten, Sackungserscheinungen und Talzuschüben. Solche Erscheinungen können leicht nachgewiesen werden, indem man in gewissen Zeitabständen vermarkte Messpunkte (Bild 1) nachkontrolliert und damit ihre Bewegung feststellt. In den meisten Fällen ergibt sich, dass sich derartige Hänge tatsächlich in einer rotationsartigen Sackung (Bild 2) bewegen. Die Geschwindigkeiten sind in der Grössenordnung von $2 \mathrm{~cm}$ (und mehr) pro Jahr. Morphologisch äussern sich diese Bewegungen in einer "wülstigen" Oberfläche des Hanges (die "Wülste" ent- sprechen langsam sackenden, sich zum Teil stauchenden Schollen des Hanges) sowie in einem charakteristischen Säbelwuchs der Bäume. Die Wirkung einer solchen Sackungsbewegung auf Gebäude kann äusserst zerstörend sein (Bild 3 ). In sehr oberflächlichen Sackungen uberschiebt sich die Rasendecke, z.T. zerreisst sie auch und ergibt so den Anblick von Rasengleiten und Kahlstellen ("Plaiken").

\section{Bergrutschungen}

Wenn die Kriechbewegung sich beschleunigt, kann sie in einen kleinen "Bergrutsch" (Bild 4) übergehen. Derartige Rutschungen sind von relativ lokaler Bedeutung, sie können sich jedoch plötzlich ereignen und gewissen Schaden erzeugen. Meistens ereignen sie sich, wenn ein an sich stabiler Hang durch natürliche oder künstliche Abtragung am Fuss an die Stabilitätsgrenze gebracht wird.

\section{Felsstürze}

Wenn die Stabilitätsgrenze eines Hanges erreicht oder überschritten wird, geschehen rapide Ereignisse. Wie schon erwähnt, kann sich die Stabilität hierbei auf die Lage einzelner Felsbrocken oder auf grosse Teile des Hanges beziehen.

Einzelne herabstuirzende Felsbrocken gehorchen den gewöhnlichen Fallgesetzen. Nach mehrmaligem Aufschlagen werden sie zerstört. Daher ist es nicht zu verwundern, dass die Brocken auf weicher Unterlage, wie Waldboden, weiter reichen als auf harter, steiniger, wo sie sehr rasch zerbrechen. Ein Wald ist daher kein Schutz vor solchen Brocken.

Grössere Felsmassen geben Anlass zu Schuttströmen, welche sich mit viel Staub und Getöse hangabwärts bewegen. Sie gehorchen den gewöhnlichen Reibungsgesetzen; ihre Reichweite kann berechnet werden.

\section{Bergstürze}

Eine Bewegung, die grosse Fels- oder Erdmassen (V $100000 \mathrm{~m} 3$ ) umfasst, wird als Bergsturz bezeichnet. Man kann sie immer noch durch das Modell der trockenen Reibung beschreiben, aber nun ist der Reibungskoeffizient eine Funktion des stürzenden Felsvolumens: Je grösser das Volumen, desto kleiner die Reibung (Bild 5). Man kann daher wiederum die Reichweite eines

Prof. Dr. A. E. Scheidegger, Institut für Geophysik der Technischen Universität, Gusshausstrasse 27 - 29, A - 1040 Wien 
erwarteten Bergsturzes berechnen, wenn das instabile Volumen abgeschätzt werden kann. Die entsprechende Formel wurde vom Schreibenden entwickelt und ist in Bild 5 graphisch dargestellt. Warum die Abnahme der Reibung mit wachsendem Volumen erfolgt, ist allerdings noch nicht ganz klar.

Das auslösende Moment eines Bergsturzes ist immer die Instabilisierung grosser Massen. Dies kann einerseits durch ein Steilerwerden des Hanges, durch Erosion am Fuss oder tektonische Hebungen, anderseits aber auch durch eine Erniedrigung der inneren Festigkeit, z.B. durch Erhöhung des Porenwasserdruckes geschehen. So wird die Füllung eines Stausees am Fuss eines Hanges sich immer nachteilig auf dessen Stabilität auswirken, was z.B. das auslösende Moment im Falle der VajontKatastrophe gewesen zu sein scheint.

Muren

Schliesslich kommen wir zu den externen Einflüssen auf Hänge. Die Schnee- und Lawinenforschung ist ein eigenes Gebiet, aber die Entstehung von Muren ("Rüfenen") ist eine logische Generalisierung der anderen Massenbewegungen an Hängen.

Bei Vermurungen kommt zu der Eigenbewegung von Massen die Wirkung des Wassers hinzu: Es bildet sich ein Gemisch von Wasser und Festmaterial; das letztere wird durch die Turbulenz des Wassers in Suspension gehalten. Zur Bildung einer Mure braucht es daher immer zweierlei: genuigend Schutt und genügend Wasser. Der Schutt sammelt sich als Folge der langsamen Kriechbewegungen der Hänge über die Jahre an; bei ergiebigen Regengüssen kann sich dann eine Mure bilden. Die Mechanik des Vorganges ist nun wenigstens in seinen Grundprinzipien erklärbar.

\section{Schutz des Lebensraumes}

Man kann die oben dargestellten rein wissenschaftlichen Erkenntnisse über mechanische Ereignisse an Hängen nun zum Schutz des entsprechenden Lebensraumes verwerten.

Man sieht, dass einem "Ereignis" immer das Erreichen der Instabilität an einem Hang vorausgeht. Insofern als tektonische Bewegungen gegeben sind, - heben sich doch auch heute die Alpen noch mit einer Geschwindigkeit von $\mathrm{mm}$ im Jahr - ist wohl an eine Beeinflussung desselben durch den Menschen nicht zu denken: Man wird sich den Tatsachen anpassen müssen.

In anderer Hinsicht jedoch kann sehr wohl eine Beeinflussung stattfinden. Erosion wird durch
Abholzen beschleunigt. Verbauungen am Fuss eines Hanges verhindern die seitliche Untergrabung von Hängen durch Bergbäche. Die mögliche Instabilisierung von Hängen durch Stauseen an deren Fuss kann abgeschätzt werden. Drainage kann dem durch Regenfälle erzeugten Porenwasserdruck entgegenwirken. Schliesslich können Prognosen für katastrophale Ereignisse, wie Murgänge und Bergstürze gemacht werden: Durch eine rechtzeitige Warnung kann, wenn das Ereignis auch nicht zu verhindern ist, doch das Leben der Bevölkerung geschützt werden.

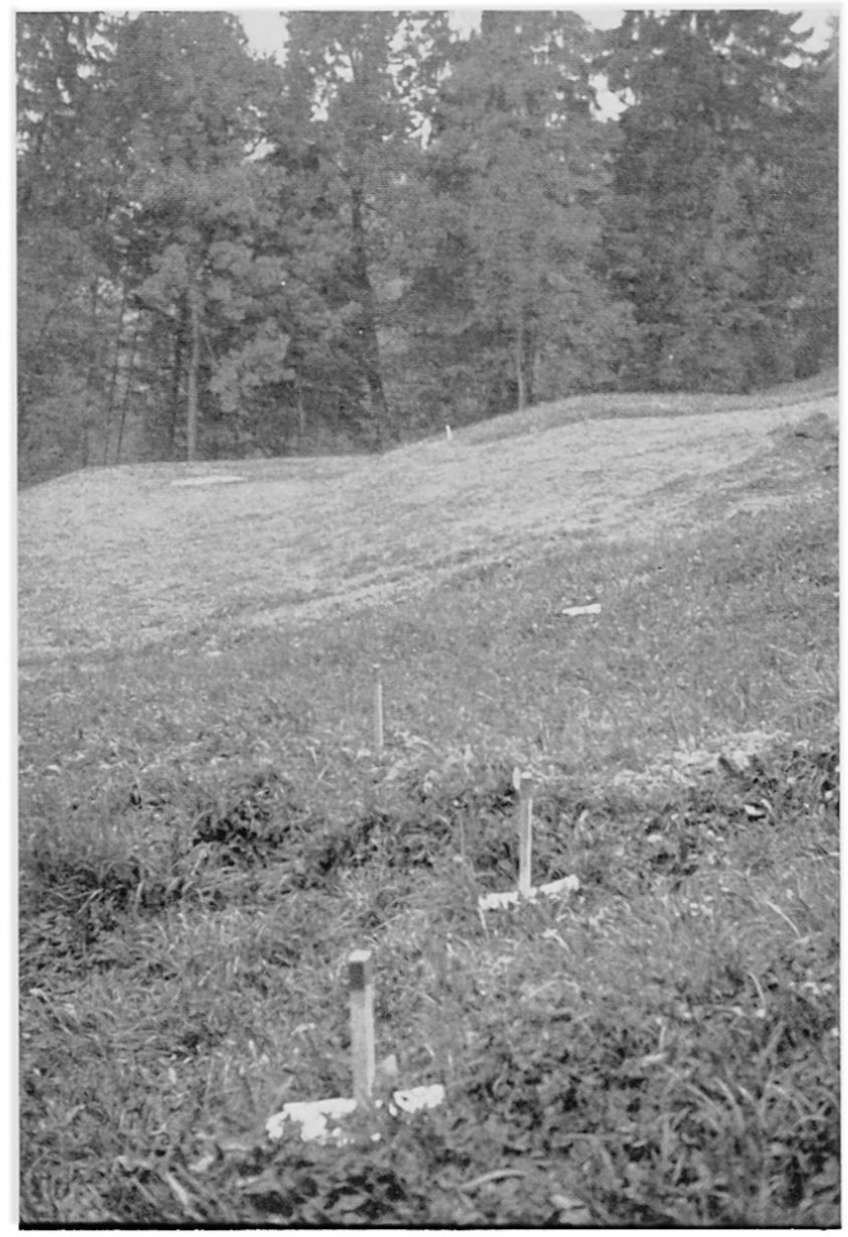

Bild 1

Nachkontrolle einer Vermarkung nach einiger Zeit: Die Pfähle waren ursprünglich in einer Geraden. 


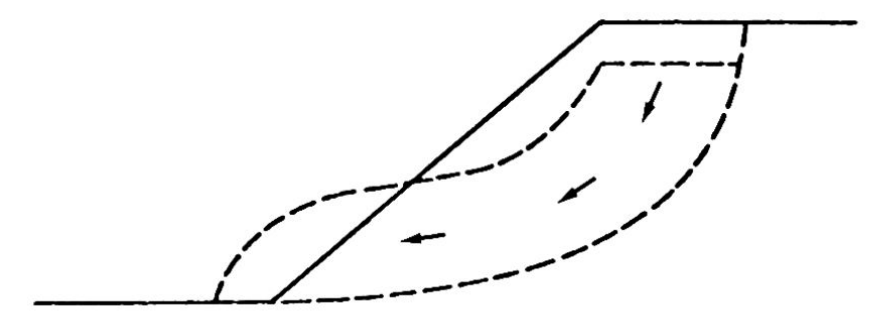

Bild 2

Schematische Darstellung einer Sackung.

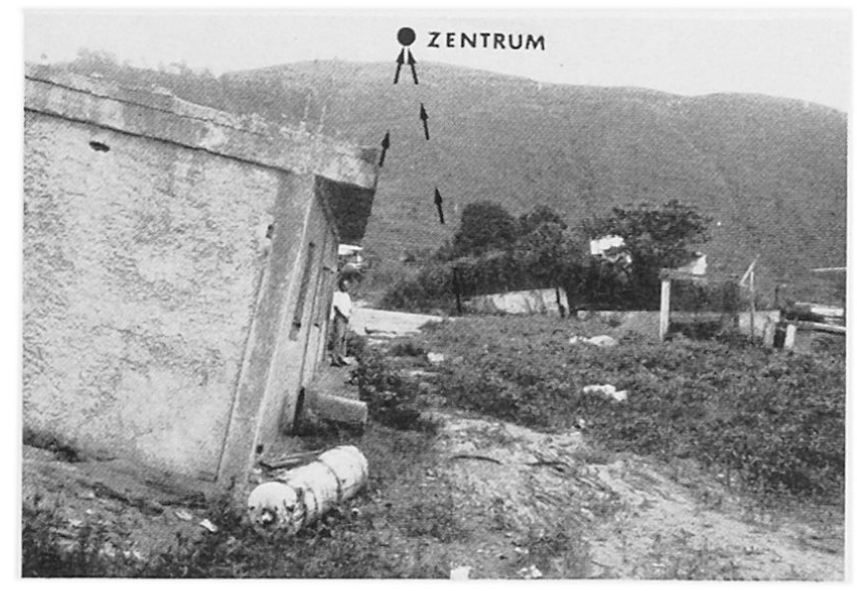

Bild 3

Rotationsförmige Sackung und Wirkung auf Bauten (bei Caracas, Venezuela).

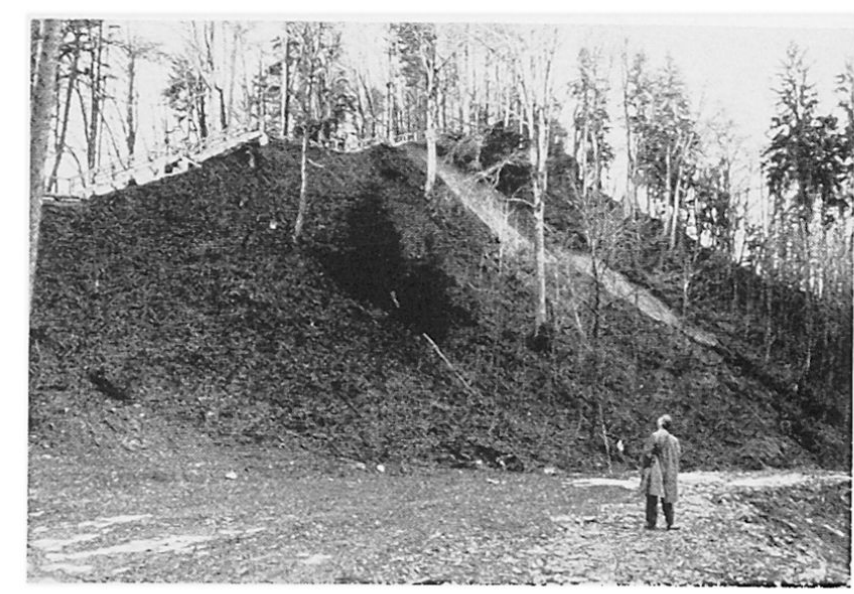

Bild 4

Durch Forststraße induzierter Bergrutsch (am Üetliberg-Kulm bei Zürich).

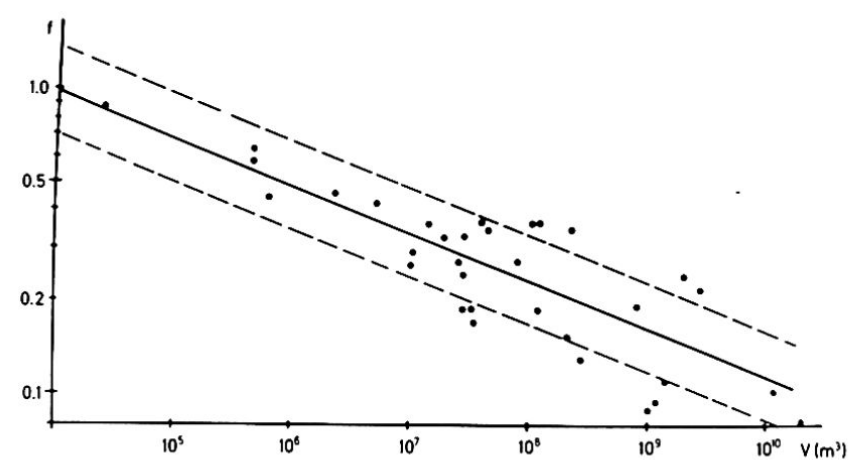

Bild 5

Korrelation zwischen Reibungskoeffizient $f$ und Bergsturzvolumen V (nach Scheidegger). 